\title{
ZUM LEXIKALISCHEN UND SEMANTISCHEN SPRACHWANDEL DER DEUTSCHEN UND POLNISCHEN RECHTSSPRACHE AM BEISPIEL DES STRAFRECHTLICHEN FACHWORTSCHATZES
}

\author{
KATARZYNA SIEWERT-KOWALKOWSKA, Dr. \\ Uniwersytet Kazimierza Wielkiego w Bydgoszczy \\ Instytut Neofilologii i Lingwistyki Stosowanej \\ ksiewert@ukw.edu.pl
}

\begin{abstract}
Der Artikel ist der Frage der Veränderungen gewidmet, die u. a. unter dem Einfluss der dynamischen politischen und sozialen Situation in der Rechtssprache erfolgen. Im Artikel werden lexikalische und semantische Veränderungen präsentiert, die sich im Laufe der Jahrhunderte im Fachwortschatz des polnischen und des deutschen Strafrechts vollzogen haben. Die Analyse wird am Beispiel der ausgewählten polnischen und deutschen Rechtstermini und -ausdrücke durchgeführt.
\end{abstract}

Schlagwörter: lexikalischer Sprachwandel, semantischer Sprachwandel, Rechtssprache, Fachwortschatz, Strafrecht

\section{O LEKSYKALNYCH I SEMANTYCZNYCH ZMIANACH JEZZYKOWYCH W NIEMIECKIM I POLSKIM JĘZYKU PRAWA NA PRZYKLADZIE SLOWNICTWA PRAWA KARNEGO}




\title{
Katarzyna Siewert-Kowalkowska: Zum Lexikalischen und ...
}

\begin{abstract}
Abstrakt: Artykuł jest poświęcony zagadnieniu zmian w języku prawa zachodzących m.in. pod wpływem zmieniającej się sytuacji politycznej i społecznej. W artykule zostaną przedstawione zmiany leksykalne i znaczeniowe, jakie dokonały się na przestrzeni wieków w słownictwie specjalistycznym polskiego i niemieckiego prawa karnego. Analiza zostanie przeprowadzona na przykładzie wybranych polskich i niemieckich terminów i wyrażeń prawnych.
\end{abstract}

Słowa klucze: zmiany leksykalne, zmiany znaczeniowe, język prawa, słownictwo specjalistyczne, prawo karne

\section{LEXICAL AND SEMANTIC LANGUAGE CHANGES OF GERMAN AND POLISH LAW LANGUAGE ON THE BASIS OF THE CRIMINAL VOCABULARY}

\begin{abstract}
The article is devoted to the problem of the changes taking place in legal language, among others under the influence of the dynamic political and social situation. The paper will present lexical and semantic changes that have taken place over the centuries in the specialized vocabulary of the Polish and German criminal law. The analysis will be carried out on the example of selected Polish and German legal terms and expressions.
\end{abstract}

Key words: lexical language changes, semantic language changes, law language, specialized vocabulary, criminal law

\section{Einleitung}

Im Vorwort der ersten Ausgabe der Zeitschrift für Anwaltspraxis (ZAP), die im April 1989 erschienen ist, konnte man folgenden Einleitungssatz lesen: „Unser Recht ist kein starres Gebilde, sondern ein Spiegelbild der Entwicklung unserer Gesellschaft“. Auch heute, nach 27 Jahren, hat diese Feststellung noch Geltung, weil Recht auf die wirtschaftlichen und sozialen Entwicklungen reagiert und im engsten Kontext mit Politik, Ideen und Zeitgeist steht. Rainer Schröder hat dies anschaulich zum Ausdruck gebracht: „Oft ist Recht gefrorene Politik“ (Schröder 2015: 100) ${ }^{1}$. Außerdem haben die

\footnotetext{
${ }^{1}$ Wie sich die Politik auf die juristische Fachsprache auswirkt, hat Michael Stolleis (1972) in seinem Artikel über die Entwicklung des Begriffs Gemeinschaft im nationalsozialistischen Deutschland und über seine anschließende Einführung in die juristische Terminologie der einzelnen Rechtsgebiete in der Zeit zwischen 1933 und 1945 dargestellt.
} 


\section{Comparative Legilinguistics 29/2017}

Weiterentwicklung von Wissenschaft und Technik, die Erschließung neuer Wissensgebiete sowie andere Ethik- und Moralvorstellungen Auswirkungen auf Recht: Neue Gesetze sind notwendig, andere müssen teilweise geändert werden und manchmal über Jahre geltende Vorschriften werden ersatzlos aufgehoben (vgl. ZAP). Mit dem Recht wandelt sich auch seine Sprache. Die Rechtssprache ist wie jede natürliche Sprache dem Prozess des Sprachwandels unterworfen, der mit dem Wandel der Welt und der Menschen im Zusammenhang steht. Sprache existiert, um es mit den Worten von Peter von Polenz zu sagen, „nur im Zeitablauf“ und sie ,ist in hohem Grade immer wieder ein Neuvollzug, bei dem selbst das schon oft Gesagte meist anders gesagt wird“ (Polenz 2009: XI). Schon diese grundlegende Eigenschaft der Sprache ist der Grund dafür, dass sich jede Sprache ständig verändert (vgl. Polenz 2009: XI). Durchschnittliche Sprachteilhaber bemerken gewöhnlich den Sprachwandel nicht, weil sie die Sprache als gegenwärtiges Kommunikationsmittel betrachten. Deshalb erscheint ihnen die Sprache unveränderlich. Nur diejenigen, die sich an den alten Sprachgebrauch erinnern können oder die mit Sprachdokumenten aus der Vergangenheit zu tun haben, können den Sprachwandel erkennen (vgl. Polenz 2009: XI).

In jeder natürlichen Sprache werden Veränderungen insbesondere in ihrem Wortschatz deutlich sichtbar (vgl. Schippan 2002: 241):

- Es werden neue Wörter gebildet, die entweder Lücken im Wortschatz ausfüllen oder andere Wörter verdrängen. In der Sprache erscheinen Neologismen, die mit der Zeit zu Wortschatzelementen werden oder die bald wieder aus dem Sprachgebrauch verschwinden.

- Im Laufe der Zeit rücken Wörter an die Peripherie des Wortschatzes, sie veralten und sterben aus, d. h. sie sind den Sprachteilhabern nicht mehr bekannt. Im gegenwärtigen Sprachgebrauch leben sie noch als Archaismen und Historismen, sie werden als alt empfunden und genutzt.

- Wörter werden aus anderen Sprachen übernommen. Diese Entlehnungen passen sich der jeweiligen Sprache an oder behalten die Merkmale ihrer Herkunftssprachen. Mit der Zeit können Fremdwörter durch einheimische ersetzt werden.

- Wörter erscheinen in neuen Kontexten, neuen Distributionen. Ihre Bedeutungen verändern sich mit Veränderungen des Gebrauchs. 
- Und schließlich verändern Wörter ihre Gebrauchssphäre: Sie gehen in einen anderen Wortschatzbereich, in eine andere Varietät der Sprache, über und ändern ihren Stellenwert im Sprachsystem.

Im Fachwortschatz der deutschen und polnischen Rechtssprache vollziehen sich vor allen die vier ersten Arten der Veränderungen, und zwar lexikalische und semantische Veränderungen. Sie zeugen davon, dass die Rechtssprache lebt und auf den in der Gesellschaft stattfindenden Wandel reagiert. Im vorliegenden Artikel werden lexikalische und semantische Veränderungen anhand des Fachwortschatzes des deutschen und polnischen Strafrechts präsentiert. Das Strafrecht wurde bewusst ausgewählt, weil sich in diesem Rechtsgebiet der Einfluss von Zeit, Ideen und Tätigkeit des Menschen widerspiegelt, d. h. das Strafrecht gilt als Spiegel der Entwicklung einer Gesellschaft und seiner Kultur: „Das Strafrecht ist Produkt und charakteristischer Spiegel der Daseinsweise und der Werte der Gesellschaft, die es formuliert" (Domenico Pulitanò zitiert nach Basile 2015: 108).

\section{Neologismen im strafrechtlichen Fachwortschatz}

Neologismen werden in der Linguistik als neu eingeführte oder neuartig gebrauchte sprachliche Ausdrücke definiert (vgl. Bußmann 2008: 470). Im Hinblick auf die Rechtssprache spricht Maciej Zieliński (2012: 190) in zweifacher Hinsicht: Neologismen der Rechtssprache können etwas Neues im Vergleich zur Gemeinsprache oder im Vergleich zur bisher verwendeten Gesetzessprache einführen. Auf dieser Grundlage unterschiedet der Verfasser drei Arten von Neologismen, die in der Rechtssprache vorkommen können: Neosemantismen (Ausdrücke, die neue Bedeutungen erhalten), Neulexeme (sie entstehen durch Wortbildung oder durch Wortschöpfung) und phraseologische Neologismen (neue Wortverbindungen, die in der Gemeinsprache nicht bekannt sind) (vgl. Zieliński 2012: 191). Als Beispiele für die erste Gruppe können solche Termini des polnischen Strafrechts genannt werden wie dokument 
(Art. $115 \S 14$ poln. StGB), młodociany (Art. $115 \S 10$ poln. StGB), przedmiot (Art. $115 \S 9$ poln. StGB) und im deutschen Strafrecht die Termini Angehöriger (§ 11 Abs. 1 Ziff. 1 StGB), Amtsträger (§ 11 Abs. 1 Ziff. 2 StGB), Maßnahme (§ 11 Abs. 1 Ziff. 8 StGB).

Neulexeme treten in modernen Rechtstexten verhältnismäßig selten auf (vgl. Zieliński 2012: 191). Die hier zwei angeführten Beispiele stammen deshalb aus der heutigen polnischen und der deutschen Strafrechtslehre: cyberprzestępczość und Internetkriminalität.

Und schließlich können zur Gruppe phraseologischer Neologismen folgende Ausdrücke des polnischen und des deutschen Strafrechts gerechnet werden: groźba bezprawna, list żelazny, stan wyższej konieczności, wina umyślna, zatarcie skazania, zbieg przestepstw; zeitige Freiheitsstrafe, sicheres Geleit, Maßregeln der Besserung und Sicherung, Strafaussetzung zur Bewährung, politische Verdächtigung, Verwarnung mit Strafbehalt.

Eine interessante Geschichte haben der deutsche Terminus Rowdytum und der polnische Terminus chuligański charakter czynu (dt. rowdyhafter Charakter der Tat), die ursprünglich entsprechend zu Neulexemen und zu phraseologischen Neologismen gerechnet werden konnten. Beide Termini stammen aus den Strafgesetzbüchern, die in der DDR und in der Volksrepublik Polen galten. Sie wurden gebildet, um den aus der Sowjetunion übernommenen Tatbestand Chuliganstvo in der deutschen und in der polnischen Rechtssprache zu benennen, und zwar um eine terminologische Lücke zu schließen. Dieser Tatbestand kann übrigens als ein typisches Institut des sozialistischen Strafrechts betrachtet werden, weil er von fast allen sozialistischen Staaten übernommen wurde (vgl. Schroeder 1983: 94).

In $\S 215$ Abs. 1 des Strafgesetzbuches der DDR von 1968 war der Straftatbestand Rowdytum definiert, wie folgt: „Wer sich an einer Gruppe beteiligt, die aus Mißachtung der öffentlichen Ordnung oder der Regeln des sozialistischen Gemeinschaftslebens Gewalttätigkeiten, Drohungen oder grobe Belästigungen gegenüber Personen oder böswillige Beschädigungen von Sachen oder Einrichtungen begeht, wird mit Freiheitsstrafe bis zu fünf Jahren oder mit Haftstrafe bestraft" (Schroeder 1983: 94). Der deutsche Terminus entstand im Wege der Wortbildung aus der englischen Entlehnung rowdy und dem deutschen Suffix -tum.

Im Rechtsbegriff Rowdytum spiegelt sich zugleich die Ideologie der damaligen Zeit wider. Im Grunde genommen könnte 
man meinen, dass dieser Tatbestand angesichts des zunehmenden Vandalismus in Form der Demolierung von Telefonzellen und öffentlichen Einrichtungen, der Verunreinigung von Parks und dergleichen ausgesprochen modern war. Allerdings insbesondere durch den unscharfen Begriff ,grobe Belästigungen gegenüber Personen" wurde Rowdytum so aufgeweicht und ausgeweitet, dass auch der politische Widerstand schnell unter diesen Tatbestand subsumiert werden konnte und der Aufrechterhaltung der sozialistischen Ordnung diente. So könnte beispielsweise die Parole mit der Komponente „Freiheit für jemanden oder für etwas“ durch linientreue Funktionäre als grobe Belästigung gegenüber Personen interpretiert und entsprechend bestraft werden (vgl. Schroeder 1983: 95). Das Rowdytum war im Strafrecht der DDR keine harmlose Umschreibung für rücksichtloses Verhalten von Beschuldigten: Selbst der Versuch war strafbar. In schweren Fällen, und zwar wenn das Gericht beispielsweise ,durch die Tat die öffentliche Ordnung oder das sozialistische Gemeinschaftsleben durch Verbreitung von Unruhe unter der Bevölkerung in besonderem Maße gefährdet" sah oder wenn es sich um einen Wiederholungstäter handelte, konnte eine Freiheitsstrafe zwischen einem und acht Jahren verhängt werden (vgl. Rath 2012).

Im polnischen Strafgesetzbuch aus dem Jahre 1969 gab es dagegen den Terminus chuligański charakter czynu (dt. rowdyhafter Charakter der Tat), der das Adjektiv chuligański enthält. Dieses wurde im Wege der Wortbildung aus der russischen Entlehnung Chuliganstvo und dem polnischen Suffix -ski gebildet. Der Rechtsbegriff wurde aus dem sowjetischen in das polnische Strafrecht mit dem Gesetz über die Verschärfung der strafrechtlichen Verantwortlichkeit für Rowdytum vom 22.05.1958 eingeführt (GBl. $\mathrm{Nr} 34$ Pos. 152) (poln. ustawa z 22 maja 1958 r. o zaostrzeniu odpowiedzialności karnej za chuligaństwo). Anschließend wurde der Begriff im damaligen Strafgesetzbuch von 1969 definiert, und zwar in Art. $120 \S 14$. Im Vergleich zum deutschen Tatbestand enthielt diese Vorschrift nur eine allgemeine Strafverschärfung für Straftaten mit „rowdyhaftem Charakter": „Charakter chuligański mają występki polegające na umyślnym zamachu na bezpieczeństwo powszechne, na zdrowie, wolność, godność osobistą lub nietykalność człowieka, na organ władzy lub administracji państwowej albo na działalność instytucji państwowej lub społecznej, na porządek publiczny, albo na umyślnym niszczeniu lub uszkodzeniu mienia, jeżeli sprawca działał 


\section{Comparative Legilinguistics 29/2017}

publicznie oraz $\mathrm{w}$ rozumieniu powszechnym bez powodu lub $\mathrm{z}$ oczywiście błahego powodu, okazując przez to rażące lekceważenie podstawowych zasad porządku prawnego “2 . Erwähnenswert ist, dass dieser Rechtsbegriff im Strafgesetzbuch von 1997 ursprünglich nicht erschien und dass er erst neulich mit dem Gesetz zur Änderung des Gesetzes Strafgesetzbuch vom 16. November 2008 in einem fast identischen Wortlaut wieder eingeführt wurde (vgl. Szczekala 2008: 79).

Bei den beiden oben beschriebenen Termini mag erstaunen, dass die englischen Entlehnungen in den Rechtssprachen der sozialistischen Staaten im Sprachgebrauch waren. In diesen Staaten waren nämlich die Vertreter der Regierungsgewalt - wie man weiß für die englische Sprache nicht besonders begeistert (Rath 2012).

Ein prägnantes Zeichen unseres Zeitgeistes ist der Begriff kulturell motivierte Straftat (auch kulturelle Straftat oder kulturell orientierte Straftat genannt), der in der Strafrechtslehre infolge der Auseinandersetzung mit den Themen der kulturellen Vielfalt der zeitgenössischen Gesellschaften entwickelt wurde (vgl. Basile 2015: 29). Die massenhaften Immigrationsströme der letzten Jahrzehnte haben das Strafrecht der westeuropäischen Gesellschaften vor große Herausforderungen gestellt. Im Ankunftsland findet der Einwanderer nämlich häufig Verhaltensregeln und insbesondere Strafrechtsnormen vor, die sich von denen seines Herkunftslandes unterscheiden. Diesem Unterschied liegt in bestimmten Fällen die unterschiedliche Kultur zugrunde. Deshalb kann er eine Handlung begehen, die im Ankunftsland als Straftat gilt, in seinem Herkunftsland aber akzeptiert oder zumindest toleriert wird (vgl. Basile 2015: 15). Dazu gehören solche Straftaten wie die sog. Blutrache, die darin besteht, „mit Blut“ den Tod eines Mitglieds der eigenen Familie oder der eigenen Gruppe

2 „Einen rowdyhaften Charakter haben Vergehen, die in einem vorsätzlichen Angriff auf die allgemeine Sicherheit, auf Gesundheit, Freiheit, persönliche Würde und Unantastbarkeit des Menschen, auf ein Organ der Staatsgewalt oder

-verwaltung oder auf die Tätigkeit einer staatlichen oder sozialen Einrichtung, auf öffentliche Ordnung oder in einer vorsätzlichen Zerstörung oder Beschädigung des Vermögens bestehen, wenn der Täter in der Öffentlichkeit und ohne Grund oder aus offensichtlich belanglosem Grund im Sinne des allgemeinen Sprachgebrauchs gehandelt hat, indem er dadurch grobe Missachtung der grundlegenden Grundsätze der Rechtsordnung bekundet" (Übers. K. S.-K.). 
zu rächen, Verletzung der Schulpflicht oder Tötung zur Verteidigung der Geschlechtsehre wie beispielsweise Ehrenmorde an Frauen sowie Zwangsheirat, die seit 2011 nach $\S 237$ StGB in Deutschland strafbar ist (vgl. Basile 2015: 171-172; Grzyb 2015: 233-234). Der italienische Strafrechtler Fabio Basile (2015: 55-56) definiert den oben genannten Begriff kulturell motivierte Straftat als ,ein Verhalten, das von einer Person verwirklicht wird, die zu einer kulturellen Minderheitsgruppe gehört, und das von der Rechtsordnung der kulturellen Mehrheitsgruppe als Straftat definiert wird. Dieses Verhalten wird jedoch in der kulturellen Gruppe der handelnden Person verziehen oder als normales Verhalten akzeptiert oder belohnt oder sogar ermutigt oder zu Verpflichtung gemacht". Dabei betont der Strafrechtler im Kapitel mit dem bezeichnenden Titel „Unsere Vergangenheit - ihre Gegenwart", dass einige Straftaten, die heute besonders häufig aus kulturellen Motiven von den Einwanderern begangen werden, dieselben sind, die einst im Ankunftsland entweder als keine Straftaten galten bzw. die geduldet oder zumindest mit allgemeiner Nachsicht bewertet wurden (vgl. Basile 2015: 174). Diese Feststellung kann mit den strafrechtlichen Termini und Ausdrücken veranschaulicht werden, die aus dem juristischen Sprachgebrauch verschwunden sind oder die im Laufe der Rechtsgeschichte ihre Bedeutung verändert haben.

\section{Alterung des strafrechtlichen Fachwortschatzes}

Im mittelalterlichen deutschen Recht wie auch im mittelalterlichen polnischen Recht war Rache als Strafe bekannt: Im deutschen Recht wurde sie Fehde bzw. Blutrache und im polnischen Recht wróżda, zwada bzw. krwawa zemsta genannt (vgl. Bardach/Leśnodorski/Pietrzak 2009: 176; Creifelds 2014: 450; Hołyst 2005: 1291). Dabei handelte es sich um den Zustand der rechtmäßigen Feindschaft zwischen dem Verletzten und dem Rechtsbrecher, (vgl. Eisenhardt 1999: 74; Köbler 1990: 79; Bardach/Leśnodorski/Pietrzak 2009: 181). Durch Fehde durfte die Sippe, die über die Unversehrtheit ihrer Mitglieder wachte, Angriffe auf Ehre oder andere Rechtsgüter ihrer Angehörigen ahnden. Durch Fehde nahmen der Verletzte und 
seine Verwandtschaft bzw. die Verwandtschaft eines Erschlagenen am Täter und seinen Verwandten Rache. Der Täter wurde zum Feinde des Verletzten und seiner Sippe, so dass sie ihn ungestraft angreifen, schädigen und unter Umständen töten konnten. Die Sippenrache konnte bei Festnahme des Täters auf der sog. handhaften Tat durch sofortige Aburteilung und Vollstreckung geübt werden (vgl. Creifelds 2014: 450; Schröder 1902: 75). Handhafte Tat lag vor, wenn der Täter auf frischer Tat oder auf der Flucht nach der Tat ergriffen wurde. Die Bezeichnung hat ihren Ursprung darin, dass der Täter die Spur der Tat noch in der Hand trug, z. B. die Diebesbeute oder eine blutige Waffe (vgl. Radbruch 2001: 503). Im mittelalterlichen polnischen Recht entsprach der handhaften Tat zbrodnia uczynkowa bzw. goracy uczynek (vgl. Bardach/Leśnodorski/Pietrzak 2009: 182).

$\mathrm{Zu}$ weiteren Historismen, also zu Lexemen, die gegenwärtig genutzt werden, um über nicht mehr existente Denotate zu kommunizieren (vgl. Schippan 2002: 248), gehören auch folgende strafrechtliche Ausdrücke des polnischen und des deutschen Strafrechts, insbesondere was Strafen anbelangt: kary krwi, główszczyzna und proskrypcja (auch wywołanie genannt) sowie Fronung, Verbannung und spiegelnde Strafen.

Unter kary krwi verstand man Strafen an Leib und Leben, und zwar die Todesstrafe und Verstümmelungsstrafen (vgl. Bardach/Leśnodorski/Pietrzak 2009: 183). Główszczyzna war eine private Geldstrafe, die der Mörder den Familienangehörigen des Erschlagenen zahlen musste (vgl. Hołyst 2005: 242)). Dieser Strafe entsprach im mittelalterlichen deutschen Recht Wergeld (vgl. Köbler 1995: 464). Bei proskrypcja handelte sich dagegen um eine Strafe, die fiktiven Tod bedeutete und die über einen Ritter in seiner Abwesenheit verhängt wurde. Der Verurteilte wurde rechtlos, sein Vermögen wurde konfisziert und seine Frau wurde zur Witwe und durfte wieder heiraten. Der Rechtlose durfte ungestraft getötet werden und Hilfeleistung galt als eine Straftat (vgl. Hołyst 2005: 778). Einen ähnlichen Stellenwert im mittelalterlichen deutschen Recht hatte Acht (auch Ächtung genannt) (vgl. Creifelds 2014: 22).

Fronung bedeutete im mittelalterlichen deutschen Recht die öffentliche Beschlagnahme von Gegenständen und Grundstücken im Zuge der Zwangsvollstreckung (vgl. Köbler 2007: 155). Bei Verbannung wurde der Täter mit dem Ausschluss aus der Gemeinschaft durch Vertreibung aus dem von dieser Gemeinschaft beanspruchten Gebiet bestraft (vgl. Köbler 2007: 438). Der 
Verbannung entsprach im mittelalterlichen polnischen Recht kara wygnania, auch banicja genannt (vgl. Bardach/Leśnodorski/Pietrzak 2009: 183). Spiegelnde Strafen waren Strafen, die nach dem Talionsprinzip vollzogen wurden, d. h. dem Täter wurde ein gleichartiges Übel zugefügt, wodurch das begangene Verbrechen am Täter selbst offenbar wurde. So verlor beispielsweise der meineidige Täter die Schwurfinger und der Verleumder die Zunge (vgl. Creifelds 2014: 1237-1238; Eisenhardt 1999: 81). Im mittelalterlichen polnischen Recht sprach man von kary odzwierciedlajace (vgl. Bardach/Leśnodorski/Pietrzak 2009: 183).

Einen sprachgeschichtlich interessanten Fall stellt die Bezeichnung eines alten Strafinstruments dar, das im mittelalterlichen polnischen Recht kuna genannt wurde und das dem ursprünglichen Pranger im mittelalterlichen deutschen Recht entsprach. Kuna und Pranger bezeichneten das Halseisen, mit dem ein Täter an einen Schandpfahl gefesselt und öffentlich zur Schau gestellt wurde (vgl. Hołyst 2005: 391; Köbler 2007: 319). Später bedeutete Pranger im deutschen Recht nur den Schandpfahl und in dieser Bedeutung wurde der Ausdruck als pregierz ins polnische mittelalterliche Recht übernommen.

\section{Bedeutungswandel im strafrechtlichen Fachwortschatz}

$\mathrm{Zu}$ den ältesten deutschen Rechtswörtern, die bis heute in der deutschen Rechtssprache leben, die aber im Laufe der Rechtsgeschichte ihre Bedeutung grundlegend verändert haben, gehört der Terminus Mord (vgl. See 1964: 204). Im deutschen mittelalterlichen Recht wurde zwischen Mord und Totschlag unterschieden. Ein wesentliches und somit ein unterscheidendes Begriffsmerkmal für den Mord war die Heimlichkeit oder Verheimlichung der Begehung (vgl. Eisenhardt 1999: 77). Es handelte sich um verheimlichte Tötung, und zwar um die nach vollbrachter Tat verdeckte oder verleugnete Tötung eines Menschen (vgl. Köbler 1995: 274; Schröder 1902: 74). Ein anderes Begriffsmerkmal für den Mord konnte die „niedrige Gesinnung“ bei der Tötung eines Menschen sein. 


\section{Comparative Legilinguistics 29/2017}

Mit Totaschlag wurde dagegen die Tötung eines Menschen bezeichnet, die kein Mord war (vgl. Köbler 1995: 408). Seit dem 14. Jahrhundert qualifizierte man die Tötung ,mit Vorbedacht“ als Mord. Mord und Totschlag wurden mit verschiedenartigen peinlichen Strafen (d. h. Strafen an Leib und Leben) geahndet. Mord wurde mit dem Tode in Form des Räderns, aber auch des Hängens und Verbrennens, Totschlag hingegen mit der „ehrlichen Strafe“ des Enthauptens bestraft (vgl. Eisenhardt 1999: 77). Einem solchen Verständnis des Mordes lag eine tatstrafrechtliche Konzeption zugrunde, wo die Tat und nicht der Täter im Mittelpunkt stand. Diese Konzeption herrschte bis zum Jahre 1941, in dem mit der Gesetzesnovelle der bisherige Paragraf 211 des 1872 erlassenen Reichsstrafgesetzbuches grundlegend neu formuliert wurde. Paragraf 211 lautete ursprünglich im Jahre 1872, wie folgt: „Wer vorsätzlich einen Menschen tödtet, wird, wenn er die Tödtung mit Ueberlegung ausgeführt hat, wegen Mordes mit dem Tode bestraft". 1941 erhielt der Paragraf einen neuen Wortlaut, und zwar „(1) Der Mörder wird mit dem Tode bestraft. (2) Mörder ist, wer - aus Mordlust, zur Befriedigung des Geschlechtstriebs, aus Habgier oder sonst aus niedrigen Beweggründen, - heimtückisch oder grausam oder mit gemeingefährlichen Mitteln oder - um eine andere Straftat zu ermöglichen oder $\mathrm{zu}$ verdecken, einen Menschen tötet. (3) Ist in besonderen Ausnahmefällen die Todesstrafe nicht angemessen, so ist die Strafe lebenslanges Zuchthaus“. Mit dieser Umformulierung wurde der Übergang von einer tatstrafrechtlichen (,wegen Mordes“) zu einer täterstrafrechtlichen („Der Mörder“) Konzeption vollzogen. Für die Strafzumessung war damit nicht mehr die Tat, sondern der Täter selbst von Bedeutung (vgl. Bockelmann 1939: 3-4).

Im heutigen deutschen Recht unterscheidet man folgende Fälle der Tötung: Mord, Totschlag, Tötung auf Verlangen und fahrlässige Tötung. Paragraf 211 StGB bestimmt: ,(2) Mörder ist, wer aus Mordlust, zur Befriedigung des Geschlechtstriebs, aus Habgier oder sonst aus niedrigen Beweggründen, heimtückisch oder grausam oder mit gemeingefährlichen Mitteln oder um eine andere Straftat zu ermöglichen oder zu verdecken, einen Menschen tötet". Somit ist Mörder, wer einen Menschen töten und dabei hinsichtlich Tatmotiv, Tatausführung oder Tatzweck besonders verwerflich handelt, und zwar aus Mordlust, zur Befriedigung des Geschlechtstriebs, d. h. um sich durch die Tötung sexuell zu befriedigen oder um das Opfer sexuell zu missbrauchen, aus Habgier oder sonst aus niedrigen 
Beweggründen (z. B. Rachsucht, Blutrache). Um den Tatbestand des Mordes zu erschöpfen, muss der Täter außerdem heimtückisch (d. h. unter Ausnutzen der Arg- und Wehrlosigkeit des Opfers) oder grausam oder mit gemeingefährlichen Mitteln (z. B. Brandstiftung) handeln, was Ermöglichung oder Verdeckung einer Straftat zum Ziel hat (vgl. Creifelds 2014: 1263-1264). Paragraf 212 StGB enthält dann den Tatbestand des Totschlags: „(1) Wer einen Menschen tötet, ohne Mörder zu sein, wird als Totschläger mit Freiheitsstrafe nicht unter fünf Jahren bestraft. (2) In besonders schweren Fällen ist auf lebenslange Freiheitsstrafe zu erkennen“".

Hier sei anzumerken, dass der Rechtsbegriff Mord nicht immer mit der moralischen Kategorisierung des Vorsatzes definiert wird (vgl. Bleyl 2013). So ist es im österreichischen Strafrecht. Paragraf 75 des österreichischen Strafgesetzbuches lautet: „Wer einen anderen tötet, ist mit Freiheitsstrafe von zehn bis zu zwanzig Jahren oder mit lebenslanger Freiheitsstrafe zu bestrafen“. Somit gilt jede Art der vorsätzlichen Tötung als Mord, ohne dass zwischen, weniger gravierenden' und ,heimtückischen' Tötungen gesetzlich differenziert wird (vgl. Bleyl 2013; Svinger/Winkler 2014: 117).

Im polnischen Recht bestand die Unterscheidung zwischen Mord (poln. mord) und Totschlag (poln. zabójstwo) in der Zeit der sog. Adelsrepublik (poln. Rzeczpospolita szlachecka bzw. I Rzeczpospolita), d. h. von der Mitte des 15 . Jh. bis zur dritten Teilung Polens 1795 (vgl. Bardach/Leśnodorski/Pietrzak 2009: 32). Als Totschlag galt vorsätzliche Tötung und als Mord vorsätzliche und „schamlose, dreiste“, d. h. schwere Tötung auf einer öffentlichen Straße oder im Haus des Opfers (vgl. Naworski 2013: 58). Diese Unterscheidung wurde mit dem Magdeburger Stadtrecht auf den polnischen Gebieten übernommen. Heute unterscheidet das polnische Strafrecht zwischen dem grundlegenden Tatbestand zabójstwo und den sechs qualifizierten Straftaten, zu denen u. a. zabójstwo ze szczególnym okrucieństwem gehört, eine Straftat, die in der Gemeinsprache als morderstwo bezeichnet wird. 


\section{Entlehnungen \\ im \\ strafrechtlichen Fachwortschatz}

In der Linguistik werden unter Entlehnung Vorgang und Ergebnis der Übernahme eines sprachlichen Ausdrucks oder einer sprachlichen Struktur aus einer Fremdsprache in die jeweilige Muttersprache verstanden. Das Phänomen findet insbesondere dann statt, wenn es in der Nehmersprache keine Bezeichnung für neu entstandene Sachen oder Sachverhalte gibt (vgl. Bußmann 2008: 164). Diese Art der Veränderungen wird anhand der Fremdwörter stalking und phishing im Polnischen sowie entsprechend Stalking und Phishing im Deutschen veranschaulicht, die neulich aus dem Englischen entlehnt wurden und in der polnischen und der deutschen Strafrechtslehre gebraucht werden.

Bei Stalking (engl. nachstellen, heranpirschen) handelt es sich um das wiederholte Nachstellen und Verfolgung einer Person (vgl. Creifelds 2014: 1186). Als Straftat findet sich jedoch der Ausdruck Stalking weder im polnischen noch im deutschen Strafgesetzbuch. Stalking wurde nämlich durch einheimische Termini ersetzt und entsprechend definiert: als uporczywe nękanie in Art. 190a § 1 poln. StGB und als Nachstellung § 238 StGB (vgl. Creifelds 2014: 887; Mrozek/Golińska-Konecko 2015: 74; Staręga 2012: 191-192).

Ähnlich verhält es sich mit dem Ausdruck phishing bzw. Phishing, der mit der Online-Banking-Kriminalität im Zusammenhang steht (vgl. Mikołajczyk 2014: 104; Seidl/Fuchs 2010: 85). Unter Phishing versteht man die illegale Beschaffung von Zugangsdaten zum Online-Banking, die Identitätsdiebstahl zum Ziel hat (vgl. Creifelds 2014: 973; Mikołajczyk 2014: 108). Im deutschen Strafgesetzbuch ist Phishing als Fälschung oder Gebrauch beweiserheblicher Daten (strafbar nach \& 269 StGB) sowie als Computerbetrug (strafbar nach $\S 263 \mathrm{a} \mathrm{StGB}$ ) geregelt (vgl. Creifeld 2014: 270-271, 973). Im polnischen Strafrecht fällt diese Straftat unter die Bestimmungen des Art. 287 poln. StGB: In diesem Fall liegt Computerbetrug vor (vgl. Sobiech 2011). 


\section{Zusammenfassung}

Die präsentierte Analyse der Beispiele der ausgewählten deutschen und polnischen strafrechtlichen Termini und Ausdrücke hat ergeben, dass im Recht sprachliche, rechtliche, kulturelle und sozialpolitische Verhältnisse miteinander verknüpft waren und nach wie vor sind. So wie das Recht von der wirtschaftlichen und sozialen Entwicklung lebt, so lebt auch die Rechtssprache - wie dies Anne Lise Kjær (1995: 42) formuliert hat - „,on der Realität, in der sie verwendet wird“.

\section{Bibliographie}

Bardach, Juliusz, Bogusław Leśnodorski, und Michał Pietrzak. 2009. Historia ustroju i prawa polskiego. Warszawa: LexisNexis.

Basile, Fabio, übers. 2015. Multikulturelle Gesellschaft und Strafrecht Die Behandlung der kulturell motivierten Straftaten. Berlin: LIT Verlag.

Bleyl, Henning. 2013. Niedrige Beweggründe sollten kein Maßstab mehr sein. http://www.zeit.de/politik/deutschland/201311/rechtsprechung-gesetz-mord-paragraf-211 (Zugriff am 31.05.2016)

Bockelmann, Paul. 1939. Studien zur Täterschaftrecht. Teil 1. Berlin: de

Gruyter. https://books.google.pl/books/about/Studien_zum_T\%C3\%A4 terstrafrecht_1_1939.html?id=8jIgNQEACAAJ\&redir_esc=y (Zugriff am 29.05.2016)

Bußmann, Hadumod. 2008. Lexikon der Sprachwissenschaft. Stuttgart: Kröner.

Creifelds, Carl. 2014. Rechtswörterbuch. München: C. H. Beck. Grzyb, Magdalena. 2015. Les aspects criminologiques et pénaux des délits culturellement motivés. Dissertation, Université de Bordeaux. 
Hołyst, Brunon, red. 2005. Wielka encyklopedia prawa. Warszawa: Wydawnictwo Prawo i Praktyka Gospodarcza.

Kjær, Anne Lise. 1995. Vergleich von Unvergleichbarem. Zur kontrastiven Analyse unbestimmter Rechtsbegriffe. In Von der Allgegenwart der Lexikologie. Kontrastive Lexikologie als Vorstufe zur zweisprachigen Lexikographie, hrsg. HansPeder Kromann, Anne Lise Kjær, S. 39-56. Tübingen: Max Niemeyer.

Köbler, Gerhard. 1990. Deutsche Rechtsgeschichte. München: Vahlen.

Köbler, Gerhard. 1995. Etymologisches Rechtswörterbuch. Tübingen: J.C.B. Mohr.

Köbler, Gerhard. 2007. Juristisches Wörterbuch. München: Vahlen.

Mikołajczyk, Krzysztof. 2014. Przestępstwa związane z wykorzystaniem bankowości elektronicznej - skimming. In: Przegląd Bezpieczeństwa Wewnętrznego 10, S. 104-120.

Mrozek, Jacek Janusz, und Magdalena Golińska-Konecko. 2015. Przestępstwo stalkingu wobec osób powszechnie znanych. In Media. Kultura. Komunikacja Społeczna 2, S. 73-83.

Naworski, Zbigniew. 2013. Stosunek do życia ludzkiego w prawie karnym Rzeczypospolitej szlacheckiej (stan normatywny a praktyka). In Białostockie Studia Prawnicze 3, S. 55-64.

Polenz, Peter von. 2009. Geschichte der deutschen Sprache. Berlin. New York: Walter de Gruyter.

Radbruch, Gustav. 2001. Gesamtausgabe. Band 11. Strafrechtsgeschichte. Heidelberg: C.F. Müller.

Rath, Martin. 2012. Juristische Sprachgeschichte. Rowdy als Rechtsbegriff. In Legal Tribune Online (02.09.2012), http://www.lto.de/recht/feuilleton/f/juristische-

pprachgeschichte-rowdy-als-rechtsbegriff/ (Zugriff am 17.05.2016).

Schippan, Thea. 2002. Lexikologie der deutschen Gegenwartssprache. Tübingen: Max Niemeyer Verlag.

Schröder, Richard. 1902. Lehrbuch der deutschen Rechtsgeschichte. Leipzig: Veit \& Comp. https://archive.org/stream/lehrbuchderdeut00schr/lehrbuchder deut00schr_djvu.txt (Zugriff am 31.05.2016)

Schröder, Rainer. 2015. Lust auf Rechtsgeschichte? In: Rechtslehre. Jahrbuch der Rechtsdidaktik 2013/2014, hrsg. Bernhard Bergmans, S. 97-102. Berlin: BWV. 
Katarzyna Siewert-Kowalkowska: Zum Lexikalischen und ...

Schroeder, Friedrich-Christian. 1983. Das Strafrecht des realen Sozialismus. Eine Einführung am Beispiel der DDR. Opladen: Westdeutscher Verlag.

See, Klaus von. 1964. Altnordische Rechtswörter. Philologische Studien zur Rechtsauffassung und Rechtsgesinnung der Germanen. Tübingen: Max Niemeyer.

Seidl, Alexander, Fuchs, Katharina. 2010. Zur Strafbarkeit des Phishing nach Inkrafttreten des 41. Strafrechtsänderungsgesetzes. In HRRS Onlinezeitschrift für Höchstrichterliche Rechtsprechung zum Strafrecht 2, S. 8592. http://www.hrr-strafrecht.de/hrr/archiv/11-06/?sz=9 (Zugriff am 25.05.2016).

Sobiech, Łukasz. 2011. Za wyłudzenie danych do konta można trafić do więzienia. In Gazeta Prawna vom 15.12.2011. http://prawo.gazetaprawna.pl/artykuly/580549,za-wyludzeniedanych-do-konta-mozna-trafic-do-wiezienia.html (Zugriff am 30.05.2016).

Staręga, Marta. 2012. Stalking jako nowy czyn zabroniony w polskim kodeksie karnym. Aspekt prawny oraz znaczenie społeczne. In Zeszyty Naukowe Uniwersytetu PrzyrodniczoHumanistycznego w Siedlcach 94, S. 191-201.

Stolleis, Michael. 1972. Gemeinschaft und Volksgemeinschaft. Zur juristischen Terminologie im Nationalsozialismus. In Vierteljahrshefte für Zeitgeschichte, Jahrgang 20, Heft 1, 1638.

Svinger, Ute, und Katharina Winkler. 2014. Österreichisches Rechtswörterbuch. Wien: MANZ.

Szczekala, Agnieszka, 2008. Chuligański charakter czynu. In Prokuratura i Prawo 6, S. 76-89.

Zieliński, Maciej. 2012. Wykładnia prawa. Zasady, reguły, wskazówki. Warszawa: LexisNexis.

\section{Internetquellen}

Bundesgesetz vom 23. Jänner 1974 über die mit gerichtlicher Strafe bedrohten Handlungen (Strafgesetzbuch). 
https://www.ris.bka.gv.at/GeltendeFassung.wxe?Abfrage $=\mathrm{Bu}$ ndesnormen\& Gesetzesnummer $=10002296$

Strafgesetzbuch in der Fassung der Bekanntmachung vom 13. November $\quad 1998 . \quad h t t p: / / w w w . g e s e t z e-i m-$ internet.de/bundesrecht/stgb/gesamt.pdf (Zugriff am 04.05.2016)

Ustawa z dnia 6 czerwca 1997 r. - Kodeks karny. http://isap.sejm.gov.pl/Download; jsessionid= D9C3BC66C700683605A8A52AB38BB93D?id=WDU19970 880553\&type $=3$ (Zugriff am dostępu: 04.05.2016)

$\mathrm{ZAP}=$ Zeitschrift für die Anwaltspraxis. https://www.zapverlag.de/SYSTEM/Verlag/Wir-ueber-uns (Zugriff am 25.05.2016) 
